\title{
SOR-based alternately linearized implicit iteration method for nonsymmetric algebraic Riccati equations
}

\author{
Tongxin Yan ${ }^{1}$ and Changfeng $\mathrm{Ma}^{1}$ \\ ${ }^{1}$ Fujian Normal University
}

September 1, 2020

\begin{abstract}
In this paper, we propose a class of SOR-based alternately linearized implicit iteration method (SORALI) for computing the minimal nonnegative solution of nonsymmetric algebraic Riccati equations (NARE). Under certain conditions, we prove the convergence of the iterative method. Finally, numerical examples are given to show the iterative method is efficient.
\end{abstract}

\section{Hosted file}

SORARE.pdf available at https://authorea.com/users/355395/articles/478651-sor-basedalternately-linearized-implicit-iteration-method-for-nonsymmetric-algebraic-riccatiequations 